Notre Dame Journal of Formal Logic

Volume 32, Number 4, Fall 1991

\title{
A Multidimensional Modal Translation for a Formal System Motivated by Situation Semantics
}

\author{
JUAN BARBA ESCRIBA
}

\begin{abstract}
The content of this paper is as follows: first a multidimensional quantified modal logic is presented, and soundness, completeness, and compactness are proven for it. Then we propose a translation of the sentences of the language $\mathbb{L}$ presented in J. Barba's "Two formal systems for situation semantics" (to appear in Notre Dame Journal of Formal Logic) and prove that it is a suitable translation. Finally, we propose a notion of persistence for the sentences of $\mathbb{L}$ (inspired in the concept of persistence defined in Situation Semantics) and find its modal counterpart in our multidimensional system.
\end{abstract}

\section{A multidimensional modal system}

The language $\mathbf{L}(M) \quad \mathbb{L}(M)$ is a multidimensional quantified modal language whose logical symbols are $\wedge, \neg, \square, \forall, E$ ! (the existence predicate), =, and $\otimes_{i}$, for each natural number $i$. We also have predicate, constant, and variable symbols. The set of wffs contains only closed formulas and is defined in the standard way.

The semantics for $\mathbb{L}(M)$ is as follows: $\mathbb{L}(M)$ 's models are structures $M=$ $\langle\mathbb{W}, \mathbb{U}, \mathbb{D}, I, R\rangle$, where $\mathbb{W}$ and $\mathbb{U}$ are sets, $\mathbb{W} \neq \varnothing, \mathbb{D}$ is a function defined on $\mathbb{W}$ such that $\mathbb{D}(w) \subseteq \mathbb{U}, I$ is an interpretation function such that $I(c) \in \mathbb{U}$, for each constant $c$ and $I(P, w) \subseteq \mathbb{U}^{n}$, for each $n$-ary predicate $P$ and $w \in \mathbb{W}$. $R$ is a reflexive and transitive binary relation on $\mathbb{W}$. We further stipulate that, for every $v, w \in \mathbb{W}$, if $v R w$ then $\mathbb{D}(v) \subseteq \mathbb{D}(w)$ (nested domains condition).

We shall use $\mathbb{U}$-formulas. A $\mathbf{U}$-formula is the result of substituting elements of $\mathbb{U}$ for some constants in a wff. (The concept of $\mathbb{U}$-formula has been borrowed from Smullyan [6]. Notice, however, that this concept is sound only if we assume that no member of $\mathbb{U}$ is already a constant or a variable of our language. This is a harmless assumption, but it is necessary, as the referee pointed out to me.) A parameter of a $\mathbb{U}$-formula $A$ is either a constant or an element $r$ 
of $\mathbb{U}$ in $A$. We extend the function $I$ in such a way that it is defined for any parameter $t$, stipulating that for any $r \in \mathbb{U}, I(r)=r$.

We can now define a valuation function $V$ which evaluates $\mathbb{U}$-formulas in a model $M$ relative to $\omega$-tuples $\vec{u}$ in $\mathbb{W}$ and an element $u_{i}$ in $\vec{u}$ as follows:

- For any $\mathbb{U}$-wff $A$, $\omega$-tuple $\vec{u}$ in $\mathbb{W}$ and $u_{i}(i \geq 0), V\left(A, \vec{u}, u_{i}\right) \in\{1,0\}$.

- $V\left(t=t^{\prime}, \vec{u}, u_{i}\right)=1$ iff $I(t)=I\left(t^{\prime}\right)$.

- $V\left(P t_{1}, \ldots, t_{n}, \vec{u}, u_{i}\right)=1$ iff $\left\langle I\left(t_{1}\right), \ldots, I\left(t_{n}\right)\right\rangle \in I\left(P, u_{i}\right)$.

- $V\left(\mathrm{E} ! t, \vec{u}, u_{i}\right)=1$ iff $I(t) \in \mathbb{D}\left(u_{i}\right)$.

- $V\left(\neg A, \vec{u}, u_{i}\right)=1$ iff $V\left(A, \vec{u}, u_{i}\right)=0$.

- $V\left(A \wedge B, \vec{u}, u_{i}\right)=1$ iff $V\left(A, \vec{u}, u_{i}\right)=V\left(B, \vec{u}, u_{i}\right)=1$.

- $V\left(\forall x A, \vec{u}, u_{i}\right)=1$ iff $V\left(A[r / x], \vec{u}, u_{i}\right)=1$ for every $r \in \mathbb{U}$.

- $V\left(\otimes_{j} A, \vec{u}, u_{i}\right)=1$ iff $V\left(A, \vec{u}, u_{j}\right)=1$.

- $V\left(\square A, \vec{u}, u_{i}\right)=1$ iff $V(A, \vec{u}[v / i], v)=1$, for every $v \in \mathbb{W}$ such that $u_{i} R v$, where $\vec{u}[v / i]$ is the result of substituting $v$ for $u_{i}$ in $\vec{u}$.

As the accessibility relation $R$ is a reflexive and transitive relation, we have a logic whose propositional basis is $S 4$. The quantification domain is $\mathbb{U}$, but combining quantifiers with the existence predicate $E$ ! and operators $\otimes_{i}$ we can quantify over the domain of any $u_{i}$ in $\vec{u}$ using formulas of the following kind:

$$
\forall x\left(\otimes_{i} \mathrm{E} ! x \rightarrow A(x)\right) .
$$

We say that a sentence $A$ is valid in a model $M$ iff for every $\vec{u}$ in $M$, $V\left(A, \vec{u}, u_{0}\right)=1$. $A$ is valid iff it is valid in any model $M$.

Let $\Sigma, \Omega$ be sets of wffs of $\mathbb{L}(M) .\langle\Sigma, \Omega\rangle$ is verified by a model $M$ iff for every $\vec{u}$ in $M$, if $V\left(A, \vec{u}, u_{0}\right)=1$ for every $A \in \Sigma$, then $V\left(B, \vec{u}, u_{0}\right)=1$ for some $B \in \Omega$, and otherwise it is refuted by $M .\langle\Sigma, \Omega\rangle$ is valid iff it is verified by every model $M$. Whenever a pair $\langle\Sigma, \Omega\rangle$ is valid, we write $\Sigma \vDash_{M} \Omega$. (We omit the index $M$ when no confusion can arise.)

Indices We shall use a certain kind of indices as an auxiliary device in the formulation of a deductive system for $\mathbb{L}(M)$ and in the completeness proof. (Our use of indices is inspired in the ideas contained in Fitting [3], Smullyan [7], and, particularly, Marraud [4] and [5]). We use $a, a^{\prime}, a_{1}, \ldots$ to represent indices. Each index has the following structure:

$$
a=\left\langle\left\langle a_{0}, \ldots, a_{i}, \ldots\right\rangle, a_{i}\right\rangle
$$

(or, more briefly, $a=\left\langle\vec{a}, a_{i}\right\rangle$ ), where, for each $j \geq 0 a_{j}$ is a finite sequence of natural numbers whose first term is $j$. Let $k$ be a sequence (perhaps empty) of natural numbers. By $a_{j} \wedge k$ we mean the result of adding the sequence $k$ on the right of $a_{j} . a_{j} \wedge k$ is then an extension of $a_{j}$. If $k \neq \varnothing$, the extension is proper. For further use, let $\vec{o}=\langle\langle 0\rangle,\langle 1\rangle,\langle 2\rangle \ldots\rangle$, and let $o$ be the index $\langle\vec{o},\langle 0\rangle\rangle$.

Index readings An index reading in a model $M$ is a function $f$ defined on the set of all finite sequences of natural number and whose range is a subset of W. Every index reading must satisfy the following restriction:

For any two sequences $s$ and $s^{\prime}$ such that $s^{\prime}$ is an extension of $s, f(s) R f\left(s^{\prime}\right)$. We write $f(\vec{a})$ for $\left\langle f\left(a_{0}\right), \ldots, f\left(a_{i}\right), \ldots\right\rangle$ and $f(a)$ for $\left\langle f(\vec{a}), f\left(a_{i}\right)\right\rangle$. 
Valuation of indexed formulas An indexed formula is a pair $\langle A, a\rangle$, where $A$ is a wff and $a$ is an index. We shall write $A_{a}$ instead of $\langle A, a\rangle$. We can define a valuation function defined on indexed formulas relative to an index reading $f$ as follows:

$$
V\left(A_{a}, f\right)=V\left(A, f(\vec{a}), f\left(a_{i}\right)\right) .
$$

An index reading $f$ verifies an indexed formula $A_{a}$ in $M$ iff $V\left(A_{a}, f\right)=1$. Notice that $A$ is valid in a model $M$ iff for every index reading $f, V\left(A_{0}, f\right)=1$.

The sequent system $S(M) \quad$ A sequent is a pair $\langle\Gamma, \Delta\rangle$, where $\Gamma$ and $\Delta$ are finite sets of wffs of $\mathbb{L}(M)$. An indexed sequent is a pair $\langle\Gamma, \Delta\rangle$ where $\Gamma, \Delta$ are sets of indexed formulas. All sequents and formulas appearing in the system $S(M)$ are indexed ones, so $S(M)$ is a proof system for indexed sequents. Let $\langle\Gamma, \Delta\rangle$ be an indexed sequent. We write $\Gamma \vdash \Delta$ to mean that $\langle\Gamma, \Delta\rangle$ is provable in $S(M)$.

Let $\Sigma$ be a set of wffs of $\mathbb{L}(M)$ and let $a$ be any index. Then we stipulate that $\Sigma_{a}=\left\{A_{a}: A \in \Sigma\right\}$.

Let $\Gamma, \Delta$ be sets of wffs of $\mathbb{L}(M)$ (i.e., they are sets of nonindexed formulas). We define that $\langle\Gamma, \Delta\rangle$ is provable in $S(M)(\Gamma \vdash \Delta)$ iff $\Gamma_{o} \vdash \Delta_{o}$, where $o$ is the index specified above.

$S(M)$ has the following axiom schemes and rules:

\section{Axiom schemes}

1. $\left(P c_{1}, \ldots, c_{n}\right)_{a} \vdash\left(P c_{1}, \ldots, c_{n}\right)_{a^{\prime}}$, provided that $a=\left\langle\vec{a}, a_{i}\right\rangle$ and $a^{\prime}=$ $\left\langle\vec{a}^{\prime}, a_{i}\right\rangle$.

2. $\left(t_{1}=s_{1}\right)_{a 1}, \ldots,\left(t_{n}=s_{n}\right)_{a n},\left(P t_{1}, \ldots, t_{n}\right)_{a} \vdash\left(P s_{1}, \ldots, s_{n}\right)_{a^{\prime}}$, where $a$ and $a^{\prime}$ are as in scheme 1 .

3. $\left(c=c^{\prime}\right)_{a} \vdash\left(c=c^{\prime}\right)_{a^{\prime}}$, for any indices $a$ and $a^{\prime}$.

Rules $\quad(\Gamma, \Delta$ are sets of indexed formulas).

W $\frac{\Gamma \vdash \Delta}{\Gamma, \Gamma^{\prime} \vdash \Delta, \Delta^{\prime}}$

1a $\frac{\Gamma, A_{a} \vdash \Delta}{\Gamma \vdash \neg A_{a}, \Delta}$

1b $\frac{\Gamma \vdash A_{a}, \Delta}{\Gamma, \neg A_{a} \vdash \Delta}$

2a $\frac{\Gamma, A_{a}, B_{a} \vdash \Delta}{\Gamma, A \wedge B_{a} \vdash \Delta}$

2b $\frac{\Gamma \vdash A_{a}, \Delta ; \Gamma \vdash B_{a}, \Delta}{\Gamma \vdash A \wedge B_{a}, \Delta}$

3a $\frac{\Gamma, A(t)_{a} \vdash \Delta}{\Gamma, \forall x A(x)_{a} \vdash \Delta}$

3b $\frac{\Gamma \vdash A(t)_{a}, \Delta}{\Gamma \vdash \forall x A(x)_{a}, \Delta}$

(*) provided $t$ does not occur in $\forall x A(x), \Gamma, \Delta$.
4a $\frac{\Gamma, A_{a^{\prime}} \vdash \Delta}{\Gamma, \otimes_{i} A_{a} \vdash \Delta}$
$(*)$
4b $\frac{\Gamma \vdash A_{a^{\prime}}, \Delta}{\Delta \vdash \otimes_{i} A_{a}, \Delta}$

(*) provided $a^{\prime}=\left\langle\vec{a}, a_{i}\right\rangle$ and $a=\left\langle\vec{a}, a_{j}\right\rangle$. 

5a $\frac{\Gamma, A_{a^{\prime}} \vdash \Delta}{\Gamma, \square A_{a} \vdash \Delta}$
5b $\frac{\Gamma \vdash A_{a^{\prime}}, \Delta}{\Gamma \vdash \square A_{a}, \Delta} \quad$ (**)

(*) provided $a=\left\langle\left\langle a_{0}, \ldots, a_{i}, \ldots\right\rangle, a_{i}\right\rangle$ and $a^{\prime}=\left\langle\left\langle a_{0}, \ldots, a_{i}^{\prime}, \ldots\right\rangle, a_{i}^{\prime}\right\rangle$, where, for some finite sequence of natural numbers $k, a_{i}^{\prime}=a_{i} \wedge k$.

(**) $a$ and $a^{\prime}$ are as in the former case and, moreover, there is no index in $\Gamma, \Delta$ containing a sequence $a_{i}^{\prime \prime}$ such that, for some sequence $k, a_{i}^{\prime \prime} \wedge k=a_{i}^{\prime}$ or $a_{i}^{\prime \prime}=a_{i}^{\prime} \wedge k$.
6a $\frac{\Gamma, c=c_{a}^{\prime} \vdash \Delta}{\Gamma, c^{\prime}=c_{a} \vdash \Delta}$
6b $\frac{\Gamma \vdash c=c_{a}^{\prime}, \Delta}{\Gamma \vdash c^{\prime}=c_{a}, \Delta}$
$7 \frac{\Gamma, c=c_{a}^{\prime \prime} \vdash \Delta}{\Gamma, c=c_{a 1}^{\prime}, c^{\prime}=c_{a 2}^{\prime \prime} \vdash \Delta}$
$8 \frac{\Gamma, c=c_{a} \vdash \Delta}{\Gamma \vdash \Delta}$

Soundness of $S(M) \quad$ Let $\Gamma, \Delta$ be (finite or infinite) sets of indexed formulas. We say that $\langle\Gamma, \Delta\rangle$ is valid in a model $M$ iff for every index reading $f$, if $V(A, f)=1$ for every $A \in \Gamma$, then for some $B \in \Delta, V(B, f)=1$. Otherwise, $\langle\Gamma, \Delta\rangle$ is refuted by $M .\langle\Gamma, \Delta\rangle$ is valid $(\Gamma \vDash \Delta)$ iff it is valid in every $M$.

Lemma 1 Let $\Gamma, \Delta$ be finite sets of indexed formulas. Then, $\Gamma \vdash \Delta$ only if $\Gamma \vDash \Delta$.

The proof strategy is the usual one. It is immediate that all axioms have the desired property, and it is not difficult to check that rules transmit it. This is obvious for Rule W, and the proof is easy for Rules 1, 2, 3, 4, 6, 7, and 8. So, we shall detail only Rules $5 \mathrm{a}$ and $5 \mathrm{~b}$.

5a: Let $a$ and $a^{\prime}$ be as specified. Suppose $\Gamma, A_{a^{\prime}} \vdash \Delta$. By hypothesis, $\left\langle\Gamma \cup\left\{A_{a^{\prime}}\right\}, \Delta\right\rangle$ is valid. Applying 5a we can infer $\Gamma, \square A_{a} \vdash \Delta$. Suppose $\left\langle\Gamma \cup\left\{\square A_{a}\right\}, \Delta\right\rangle$ is not valid. Then there are a model $M$ and an index reading $f$ verifying every formula in $\Gamma \cup\left\{\square A_{a}\right\rangle$ but none in $\Delta$. Then, $V\left(\square A_{a}, f\right)=1$, i.e., $V\left(A, f(\vec{a}), f\left(a_{i}\right)\right)=1$ (where $\left.a=\left\langle\vec{a}, a_{i}\right\rangle\right)$, thus $V(A, f(\vec{a})[u / i], u)$, for every $u \in \mathbb{W}$ such that $f\left(a_{i}\right) R u$. $a_{i}^{\prime}$ is an extension of $a_{i}$, so $f\left(a_{i}\right) R f\left(a_{i}^{\prime}\right)$. Then, $V\left(A, f\left(\vec{a}^{\prime}\right), f\left(a_{i}^{\prime}\right)\right)=1$, and $V\left(A_{a^{\prime}}, f\right)=1$. But this means that $f$ refutes $\left\langle\Gamma \cup\left\{A_{a^{\prime}}\right\}, \Delta\right\rangle$ in $M$, contradicting the hypothesis.

5 b: Let $\Gamma \vdash A_{a^{\prime}}, \Delta$, as specified in Rule 5 b. Suppose $\left\langle\Gamma,\left\{\square A_{a}\right\rangle \cup \Delta\right\rangle$ is not valid; that is, for some $f$ in some $M, f$ verifies every formula $\Gamma$ but none in $\left\{A_{a}\right\} \cup \Delta$. Then, $V\left(\square A_{a}, f\right)=0$, i.e., $V\left(\square A_{a}, f(\vec{a}), f\left(a_{i}\right)\right)=0$, and for some $u \in \mathbb{W}$ such that $f\left(a_{i}\right) R u, V(A, f(\vec{a})[u / i], u)=0$. Let $g$ be an index reading defined as below:

a. $g\left(a_{i}^{\prime}\right)=u$.

b. $g\left(a_{i}\right)=f\left(a_{i}\right)$.

c. for every sequence of natural numbers $b$ which is a proper extension of $a_{i}$ and such that $a_{i}^{\prime}$ is an extension of $b$ or such that $b$ is an extension of $a_{i}^{\prime}, g(b)=u$.

d. $g(b)=f(b)$ for any sequence $b$ not considered above.

$g$ satisfies the conditions imposed on index readings: for any extension $k$ of $a_{i}$ of which $a_{i}^{\prime}$ is an extension, $g\left(a_{i}\right) R g(k)$, because $g(k)=u$ and $g\left(a_{i}\right)=f\left(a_{i}\right) R u$. 
Moreover, as $R$ is a reflexive relation, $u R u$, and so $g(k) R g\left(a_{i}^{\prime}\right)$. By the same reason, for any extension $b$ of $a_{i}^{\prime}, g\left(a_{i}^{\prime}\right) R g(b)$. And for any other sequence $k$, $f(k)=g(k)$. Then, since $f$ is an index reading, so is $g$.

Now, $g\left(\vec{a}^{\prime}\right)=f(\vec{a})[u / i]$, and since $g\left(a_{i}^{\prime}\right)=u$, we have that $V\left(A, g\left(\vec{a}^{\prime}\right)\right.$, $\left.g\left(a_{i}^{\prime}\right)\right)=0$, and so $V\left(A_{a^{\prime}}, g\right)=0$. According to the proviso added to Rule $5 \mathrm{~b}$, with respect to the indices occurring in $\Gamma \cup \Delta, g$ is identical to $f$, and so $g$ verifies every formula in $\Gamma$ but none in $\Delta$. Then, $g$ refutes $\left\langle\Gamma,\left\{A_{a^{\prime}}\right\} \cup \Delta\right\rangle$, which is not valid. Thus, if this pair is valid, so is $\left\langle\Gamma,\left\{\square A_{a^{\prime}}\right\} \cup \Delta\right\rangle$.

Theorem 2 (Soundness Theorem) Then, $\Gamma \vdash \Delta$ only if $\Gamma \vDash \Delta$.

Proof: By definition, $\Gamma \vdash \Delta$ iff $\Gamma_{o} \vdash \Delta_{o}$. By Lemma 1, $\Gamma_{o} \vdash \Delta_{o}$ only if $\left\langle\Gamma_{o}, \Delta_{o}\right\rangle$ is valid, that is, only if for any $f$ in any $M$ if $V\left(A_{o}, f\right)=1$ for every $A \in \Gamma$, then $V\left(B_{o}, f\right)=1$ for some $B \in \Delta$. This means that if $V(A, f(\vec{o}), f(0))=1$ for every $A \in \Gamma$ then $V(B, f(\vec{o}), f(0))=1$ for some $B \in \Delta$. Since this must be so for any index reading $f$, we have that for any tuple $\vec{u}$ in any $M, V\left(A, \vec{u}, u_{0}\right)=1$ for every $A \in \Gamma$ only if $V\left(B, \vec{u}, u_{0}\right)=1$ for some $B \in \Delta$, so that $\Gamma \vDash \Delta$.

Completeness of $S(M) \quad$ We are going to prove that given any two sets of wffs of $\mathbb{L}(\mathrm{M}) \Sigma, \Omega$, if $\Sigma \vDash \Omega$ then there are finite sets $\Gamma, \Delta, \Gamma \subseteq \Sigma, \Delta \subseteq \Omega$ such that $\Gamma \vdash \Delta$. In order to do so we construct a tree for $\langle\Sigma, \Omega\rangle$ and prove that if the tree closes then there exist the above-mentioned sets, and that if the tree has at least an open branch, we can construct a model $M$ which refutes $\langle\Sigma, \Omega\rangle$.

First, we extend the language with a denumerable set of new constants, and suppose fixed enumerations of this set and of $\Sigma \cup \Omega$.

Each node of the tree contains a pair $\left\langle A_{a}, i\right\rangle$, where $A_{a}$ is an indexed formula of the language extended with the new constants and $i \in\{1,0\}$. The tree is constructed step by step according to the following instructions:

Step 1: Let $A$ be the first formula in the enumeration of $\Sigma \cup \Omega$. If $A \in \Sigma$, put $\left\langle A_{o}, 1\right\rangle$ in the topmost node of the tree, and if $A \in \Omega$, put $\left\langle A_{o}, 0\right\rangle$ in the topmost node.

Step $n+1:$ In each open node consider the $n$th node of the branch, and proceed according to its form. If the node is:

1a. $\left\langle\neg A_{a}, 1\right\rangle$ : Add $\left\langle A_{a}, 0\right\rangle$.

1b. $\left\langle\neg A_{a}, 0\right\rangle$ : Add $\left\langle A_{a}, 1\right\rangle$.

2a. $\left\langle A \wedge B_{a}, 1\right\rangle$ : Add the nodes $\left\langle A_{a}, 1\right\rangle$ and $\left\langle B_{a}, 1\right\rangle$.

2b. $\left\langle A \wedge B_{a}, 0\right\rangle$ : Divide the branch in two new ones. Put $\left\langle A_{a}, 0\right\rangle$ in the first one and $\left\langle B_{a}, 0\right\rangle$ in the other.

3a. $\left\langle\forall x A(x)_{a}, 1\right\rangle$ : Add nodes $\left\langle A(c)_{a}, 1\right\rangle$, for each constant $c$ occurring in the branch and then repeat $\left\langle\forall x A(x)_{a}, 1\right\rangle$.

3b. $\left\langle\forall x A(x)_{a}, 0\right\rangle$ : Add $\left\langle A(c)_{a}, 0\right\rangle$, for the first new constant $c$ not occurring in the branch.

4a. $\left\langle\otimes_{i} A_{a}, 1\right\rangle:$ Add $\left\langle A_{a^{\prime}}, 1\right\rangle$, where $a=\left\langle\vec{a}, a_{j}\right\rangle$ and $a^{\prime}=\left\langle\vec{a}, a_{i}\right\rangle$.

4b. $\left\langle\otimes_{i} A_{a}, 0\right\rangle$ : Add $\left\langle A_{a^{\prime}}, 0\right\rangle$, where $a=\left\langle\vec{a}, a_{j}\right\rangle$ and $a^{\prime}=\left\langle\vec{a}, a_{i}\right\rangle$. 
5a. $\left\langle\square A_{a}, 1\right\rangle$ : Add a node $\left\langle A_{a^{\prime}}, 1\right\rangle$ for each $a^{\prime}$ satisfying the condition established below, and finally repeat $\left\langle\square A_{a}, 1\right\rangle$. Let $a=\left\langle\left\langle a_{0}, \ldots\right.\right.$, $\left.\left.a_{i}, \ldots\right\rangle a_{i}\right\rangle$. Then $a^{\prime}=\left\langle\left\langle a_{0}, \ldots, a_{i}^{\prime}, \ldots\right\rangle, a_{i}^{\prime}\right\rangle$, where $a_{i}^{\prime}$ is any extension of $a_{i}$ occurring in the branch.

5b. $\left\langle\square A_{a}, 0\right\rangle$ : Add $\left\langle A_{a^{\prime}}, 0\right\rangle$ where $a^{\prime}$ is as follows: if $a=\left\langle\left\langle a_{0}, \ldots\right.\right.$, $\left.\left.a_{i}, \ldots\right\rangle a_{i}\right\rangle$, then $a^{\prime}=\left\langle\left\langle a_{0}, \ldots, a_{i}^{\prime}, \ldots\right\rangle, a_{i}^{\prime}\right\rangle$, where $a_{1}^{\prime}=a_{i} \wedge k$, and $k$ is the first natural number such that neither $a_{1}^{\prime}$ nor any of its extensions occur in the branch.

6a,7. $\left\langle\left(c_{1}=c_{2}\right)_{a}, 1\right\rangle$ : First of all, add $\left\langle\left(c_{2}=c_{1}\right)_{a}, 1\right\rangle$. Then for each node $\left\langle\left(c_{2}=c_{3}\right)_{a^{\prime}}, 1\right\rangle$ occurring in the branch add $\left\langle\left(c_{1}=c_{3}\right)_{a}, 1\right\rangle$.

6b. $\left\langle c=c_{a}^{\prime}, 0\right\rangle$ : Add $\left\langle c^{\prime}=c_{a}, 0\right\rangle$, if it does not appear in the branch before.

8. Then, extend the branch with all nodes $\left\langle c=c_{o}, 1\right\rangle$ such that $c$ occurs in the branch but $\left\langle c=c_{a}, 1\right\rangle$ does not appear in the branch for any $a$.

Finally, if $A$ is the $n+1$-th formula of $\Sigma \cup \Omega$, add $\left\langle A_{o}, 1\right\rangle$ if $A \in \Sigma$, and add $\left\langle A_{o}, 0\right\rangle$ if $A \in \Omega$. After having performed the instructions above for every open branch in the tree, the $n+1$-th step is finished. below:

A branch closes iff it contains a group of nodes of one of the forms detailed

1. $\left\langle\left(P c_{1}, \ldots, c_{n}\right)_{a}, 1\right\rangle,\left\langle\left(P c_{1}, \ldots, c_{n}\right)_{a^{\prime}}, 0\right\rangle$, for $a=\left\langle\vec{a}, a_{i}\right\rangle$ and $a^{\prime}=\left\langle\vec{a}^{\prime}, a_{i}\right\rangle$.

2. $\left\langle\left(c_{1}=d_{1}\right)_{a 1}, 1\right\rangle, \ldots,\left\langle\left(c_{n}=d_{n}\right)_{a n}, 1\right\rangle,\left\langle\left(P c_{1}, \ldots, c_{n}\right)_{a^{\prime}}, 1\right\rangle,\left\langle\left(P d_{1}, \ldots\right.\right.$, $\left.\left.d_{n}\right)_{a^{\prime}}, 0\right\rangle$, for $a$ and $a^{\prime}$ as in 1 .

3. $\left\langle c=c_{a}^{\prime}, 1\right\rangle,\left\langle c=c_{a^{\prime}}^{\prime}, 0\right\rangle$, for any indices $a, a^{\prime}$.

A tree closes iff every branch in it closes.

Lemma 3 If a tree for $\langle\Sigma, \Omega\rangle$ closes, there exist finite sets $\Gamma \subseteq \Sigma, \Delta \subseteq \Omega$ such that $\Gamma \vdash \Delta$.

The proof, purely routine, is left to the reader. (Notice that the numbers in front of the instructions for the construction of the tree refer to the rules which justify them.)

Lemma 4 If a tree for $\langle\Sigma, \Omega\rangle$ does not close, then $\langle\Sigma, \Omega\rangle$ is refutable.

Proof: Suppose we have constructed a tree for $\langle\Sigma, \Omega\rangle$ and the tree has an open branch. We can construct a model $M=\langle\mathbb{W}, \mathbb{U}, \mathbb{D}, I, R\rangle$ which refutes $\langle\Sigma, \Omega\rangle$. Define $M$ as follows:

$\mathbb{W}=\left\{a_{i}: a_{i}\right.$ is a sequence occurring in some index in the branch $\}$.

Let $C$ be the set of all constants appearing in the branch. Define a relation $\approx$ in $C$ as follows: $c \approx c^{\prime}$ iff $\left\langle c=c_{a}^{\prime}, 1\right\rangle$ appears in the branch for some $a . \approx$ is an equivalence relation: Instruction 8 ensures reflexivity, while instruction $6 \mathrm{a}, 7$ guarantees symmetry and transitivity.

Let $|c|$ be the equivalence class to which $c$ belongs. Then

$$
\begin{aligned}
& \mathbb{U}=\{|c|: c \in C\} \\
& \mathbb{D}\left(a_{i}\right)=\left\{|c|:\left\langle E ! c_{a}, 1\right\rangle \text { appears in the branch, for } a=\left\langle\vec{a}, a_{i}\right\rangle\right\}
\end{aligned}
$$


$I(c)=|c|$, for each $c \in C$. For any other constant $c, I(c)$ takes any arbitrary value in $\mathbb{U}$.

$I\left(P, a_{i}\right)=\left\{\left\langle\left|c_{1}\right|, \ldots,\left|c_{n}\right|\right\rangle:\left\langle\left(P c_{1}, \ldots, c_{n}\right)_{a}, 1\right\rangle\right.$ appears in the branch, for $\left.a=\left\langle\vec{a}, a_{i}\right\rangle\right\}$.

$a_{i} R u_{a j}$ iff $a_{j}$ is an extension (proper or not) of $a_{i}$.

Define an index reading $f$ as follows:

$f\left(a_{i}\right)=a_{i}$, for each $a_{i}$ in the branch. For any sequence $k$ not appearing in the branch $f(k)$ takes any adequate value in $\mathbb{W}$.

Claim For $M$ and $f$ defined as above,

a. if $\left\langle A_{a}, 1\right\rangle$ appears in the branch, $V\left(A_{a}, f\right)=1$.

b. if $\left\langle A_{a}, 0\right\rangle$ appears in the branch, $V\left(A_{a}, f\right)=0$.

The proof, an induction on the complexity of $A$, is left to the reader.

We can now prove the lemma. Consider the index $o$ as defined above. Let $f(\vec{o})=\vec{u}$ and $f(i)=u_{i}$, for each $i \geq 0$. Suppose $A \in \Sigma$. $A$ is the $n$th formula of $\Sigma \cup \Omega$, for some $n$, and $\left\langle A_{o}, 1\right\rangle$ has been introduced in the branch at the $n$th step, by the claim above, $V\left(A_{o}, f\right)=V\left(A, \vec{u}, u_{0}\right)=1$. Similarly, for any $B \in$ $\Omega, V\left(B, \vec{u}, u_{0}\right\rangle=0$. So, $M$ refutes $\langle\Sigma, \Omega\rangle$.

Theorem 5 (Completeness theorem for $S(M)$ ) $\quad \Sigma \vDash \Omega$ only if there exist finite sets $\Gamma \subseteq \Sigma, \Delta \subseteq \Omega$ such that $\Gamma \vdash \Delta$.

Proof: Suppose $\Sigma \vDash \Omega$. A tree for $\langle\Sigma, \Omega\rangle$ closes, because otherwise $\langle\Sigma, \Omega\rangle$ would be refutable, by Lemma 4 . But then, by Lemma 3 , there exist sets $\Gamma, \Delta$ as required.

Theorem 6 (Compactness theorem) Let $\Sigma, \Omega$ be two sets of wffs of $\mathbb{L}(M)$. $\Sigma \vdash \Omega$ iff there are finite sets $\Gamma \subseteq \Sigma, \Delta \subseteq \Omega$ such that $\Gamma \vDash \Delta$.

Proof: Suppose $\Sigma \vDash \Omega$. By completeness, there are finite sets $\Gamma \subseteq \Sigma, \Delta \subseteq \Omega$ such that $\Gamma \vdash \Delta$. By soundness, $\Gamma \vDash \Delta$.

$2 \boldsymbol{A}$ translation from $\mathbf{L}$ to $\mathbf{L}(M) \quad$ We propose a translation $T$ from $\mathbb{L}$ to $\mathbb{L}(M)$ defined inductively as follows:

1a. $T\left(P c_{1}, \ldots, c_{n}\right)=\mathrm{E} ! c_{1} \wedge \ldots \wedge \mathrm{E} ! c_{n} \wedge \square \diamond P c_{1}, \ldots, c_{n}$.

1b. $T\left(\neg P c_{1}, \ldots, c_{n}\right)=\mathrm{E} ! c_{1} \wedge \ldots \wedge \mathrm{E} ! c_{n} \wedge \square \diamond \neg P c_{1}, \ldots, c_{n}$.

2a. $T\left(c=c^{\prime}\right)=\mathrm{E} ! c \wedge \mathrm{E} ! c^{\prime} \wedge c=c^{\prime}$.

2b. $T\left(\neg c=c^{\prime}\right)=\mathrm{E} ! c \wedge \mathrm{E} ! c^{\prime} \wedge \neg c=c^{\prime}$.

3. $T(\neg \neg A)=T(A)$.

4a. $T(A \wedge B)=T(A) \wedge T(B)$.

4b. $T(\neg(A \wedge B))=T(\neg A) \vee T(\neg B)$.

5a. $T(A \rightarrow B)=\neg T(A) \vee T(B)$.

5b. $T(\neg(A \rightarrow B))=T(A) \wedge T(\neg B)$.

6a. $T(A \mid B)=T(A) \wedge T(B)$.

6b. $T(\neg(A \mid B))=T(A) \wedge T(\neg B)$.

7a. $T(\forall x A)=\forall x T(A)$. 
7b. $T(\neg \forall x A)=\neg \forall x \neg T(\neg A)=\exists x T(\neg A)$.

8a. $T\left(\otimes_{i} A\right)=\otimes_{i} T(A)$.

8b. $T\left(\neg \otimes_{i} A\right)=\bigotimes_{i} T(\neg A)$.

Let $f$ be a function such that for any $\mathbb{L}(M)$-model $M=\langle\mathbb{W}, \mathbb{U}, \mathbb{D}, I, R\rangle$ yields an $\mathbb{L}$-model $f(M)=N=\langle W, U, D, \mathbb{I} \mathbb{\mathbb { 1 }}, S\rangle$ according to the following clauses:

$$
\begin{aligned}
& W=\mathbb{W}, \\
& U=\mathbb{U}, \\
& D(v)=\mathbb{D}(v), \\
& \llbracket c \rrbracket=I(c) \text { and }
\end{aligned}
$$

$S(v)=\left\{A: A\right.$ is either $\operatorname{Pr}_{1}, \ldots, r_{n}$ or $\neg P r_{1}, \ldots, r_{n}$ and $\left.V(T(A), \vec{u}, v)=1\right\}$, for any $\vec{u}$ such that $v$ is a member of $\vec{u}$. (Notice that $S(v)$ depends only on $v$, and not on the other members of $\vec{u}$, as $T(A)$ contains no operator $\left.\otimes_{j}\right)$.

Lemma $7 \quad$ Let $M$ be an $\mathbb{L}(M)$-model $M=\langle\mathbb{W}, \mathbb{U}, \mathbb{D}, I, R\rangle$ and $N$ be an $\mathbb{L}$ model $N=\langle W, U, D, \mathbb{I} \mathbb{\mathbb { 1 }}, S\rangle$ such that

a. $\mathbb{U}=U$, and

b. $I(c)=\llbracket c \rrbracket$,

for every constant $c$. Suppose $\vec{u}$ is an w-tuple $\vec{u}=\left\langle u_{0}, . ., u_{i} \ldots\right\rangle$ such that, for every $i \geq 0$,

c. $u_{i} \in \mathbb{W} \cap W$,

d. $\mathbb{D}\left(u_{i}\right)=D\left(u_{i}\right)$

e. $S\left(u_{i}\right)=\left\{A: A\right.$ is $(\neg) \operatorname{Pr}_{1}, \ldots, r_{n}$ and $\left.V\left(T(A), \vec{u}, u_{i}\right)=1\right\}$. (As before, $S\left(u_{i}\right)$ depends only on $u_{i}$ )

Then, $V(T(A), \vec{u}, v)=1$ iff $\vec{u}, v \vDash A$, for every sentence $A$ of $\mathbb{L}$, and any member $v$ of $\vec{u}$.

Proof: By induction on the complexity of $A$.

Corollary $8 \quad$ Let $M=\langle\mathbb{W}, \mathbb{U}, \mathbb{D}, I, R\rangle$ and $f(M)=N=\langle W, U, D, \mathbb{I} \rrbracket, S\rangle$. Then $V(T(A), \vec{u}, v)=1$ iff $\vec{u}, v \vDash A$, for any sentence $A$ of $\mathbb{L}$, any $\omega$-tuple $\vec{u}$ in $\mathbb{W}$ and any member $v$ of $\vec{u}$.

Proof: This corollary follows immediately from Lemma 7 and the definition of $f$.

Now, let $g$ be a function which, for each $\mathbb{L}$-model $N=\langle W, U, D, \llbracket \rrbracket, S\rangle$, yields the $\mathbb{L}(M)$-model $M=g(N)=\langle\mathbb{W}, \mathbb{U}, \mathbb{D}, I, R\rangle$ constructed as explained below:

For each $u \in W$ we define:

$$
H(u)=\{u\} \cup\left\{u_{t, 1}, u_{t, 2}\right\} \cup\left\{u_{f, 1}, u_{f, 2}\right\} .
$$

Then,

$$
\begin{aligned}
& \mathbb{W}=\bigcup_{u \in W} H(u) . \\
& \mathbb{U}=U
\end{aligned}
$$




$$
\begin{aligned}
& \mathbb{D}(u)=\mathbb{D}\left(u_{t, i}\right)=\mathbb{D}\left(u_{f, i}\right)=D(u), i=1,2 . \\
& I(c)=\llbracket c \rrbracket \\
& I(P, u)=\left\{\left\langle r_{1}, \ldots, r_{n}\right\rangle: \operatorname{Pr}_{1}, \ldots, r_{n} \in S(u)\right\} . \\
& t(u)=\left\{\left\langle r_{1}, \ldots, r_{n}\right\rangle: P r_{1}, \ldots, r_{n} \notin S(u) \text { and } \neg P r_{1}, \ldots, r_{n} \notin S(u)\right\} . \\
& I\left(P, u_{t, 2}\right)=I(P, u) \cup t(u) . \\
& I\left(P, u_{t, 1}\right)=I\left(P, u_{t, 2}\right)-\left\{\left\langle r_{1}, \ldots, r_{n}\right\rangle: \neg P r_{1}, \ldots, r_{n} \in S(u)\right\} . \\
& I\left(P, u_{f, 2}\right)=I(P, u) . \\
& I\left(P, u_{f, 1}\right)=I(P, u)-\left\{\left\langle r_{1}, \ldots, r_{n}\right\rangle: \neg P r_{1}, \ldots, r_{n} \in S(u)\right\}
\end{aligned}
$$

$R$ is the reflexive and transitive closure of the least binary relation in $\mathbb{W}$ which contains all pairs of the following forms:

$\langle u, v\rangle: u, v \in W$ and $u \sqsubseteq v$.

$\left\langle u, u_{t, i}\right\rangle,\left\langle u, u_{f, i}\right\rangle$, where $u \in W$ and $i=1,2$,

$\left\langle u_{t, i}, u_{t, j}\right\rangle,\left\langle u_{f, i}, u_{f, j}\right\rangle$, where $u \in W$ and $i, j=1,2$.

It can be easily checked that this $M$ is an $\mathbb{L}(M)$-model.

Lemma 9 Let $N=\langle W, U, D, \mathbb{I} \rrbracket, S\rangle$ and $g(N)=M=\langle\mathbb{W}, \mathbb{U}, \mathbb{D}, I, R\rangle$. Let $A$ be $P c_{1}, \ldots, c_{n}$ or $\neg P c_{1}, \ldots, c_{n}$. For any $u \in W, \vec{u}$ and $u$ in $\vec{u}, \vec{u}, u \vDash A$ (i.e., $A \in S(u))$ iff $V(\square \diamond A, \vec{u}, u)=1$.

\section{Proof:}

1. Suppose $\vec{u}, u \vDash A$.

a. $A$ is $P c_{1}, . ., c_{n}$, and so $\left\langle r_{1}, \ldots, r_{n}\right\rangle \in S(u)$, for $\llbracket c_{i} \rrbracket=r_{i}, 1 \leq i \leq n$. Take any $v \in \mathbb{W}, u R v$.

i. If $v$ is $u$, take (for instance) $u_{f, 2} \cdot\left\langle r_{1}, \ldots, r_{n}\right\rangle \in I\left(P, u_{f, 2}\right)$, then $V\left(A, \vec{u}\left[u_{f, 2} / i\right], u_{f, 2}\right)=1$ and $V(\diamond A, \vec{u}, v)=1$.

ii. If $v$ is $u_{f, j}(j \in\{1,2\}), u_{f, i} R u_{f, 2}$, and, as in $1, V\left(A, \vec{u}\left[u_{f, 2} / i\right]\right.$, $\left.u_{f, 2}\right)=1$, from which it follows that $V\left(\diamond A, \vec{u}\left[u_{f, j} / i\right], u_{f, j}\right)=1$. The same reasoning works for $u_{t, j}$.

iii. If $v$ is $w \in W, A \in S(w)$, since $u \sqsubseteq w$, and, by the argument developed in $1, V(\diamond A, \vec{u}[w / i], w)=1$.

iv. If $v$ is $w_{f, j}$ (for $w \in W$ ), we have that $u \sqsubseteq w$, and, by the argument in $2, V\left(\diamond A \vec{u}\left[w_{f, j} / i\right], w_{f, j}\right)=1$. The same argument works for $w_{t, j}$.

For any $v \in \mathbb{W}$ such that $u R v, v$ must be comprehended in one of the cases considered above, thus $V(\diamond A, \vec{u}[v / i], v)=1$, and so $V(\square \diamond A, \vec{u}, u)=1$. b. Suppose now that $A$ is $\neg P c_{1}, \ldots, c_{n}$ and $\neg P r_{1}, \ldots, r_{n} \in S(u)$. Recall that $\left\langle r_{1}, \ldots, r_{n}\right\rangle \notin I\left(P, u_{t, 1}\right)$ (Idem for $\left.I\left(P, u_{f, 1}\right)\right)$, therefore

$$
\begin{gathered}
V\left(P c_{1}, \ldots, c_{n}, \vec{u}\left[u_{t, 1} / i\right], u_{t, 1}\right)=0, \quad \text { and } \\
V\left(\neg P c_{1}, \ldots, c_{n}, \vec{u}\left[u_{t, 1} / i\right], u_{t, 1}\right)=1
\end{gathered}
$$

Using this fact, we can argue cases $\mathrm{i}-\mathrm{iv}$ as above.

Regardless, if $u R v$, then $V(\diamond A, \vec{u}[v / i], v)=1$. Thus, $V(\square \diamond A, \vec{u}, u)=1$. 
2. Suppose $\vec{u}, u \# A$ :

a. $A$ is $P c_{1}, \ldots, c_{n}$. Let $\llbracket c_{j} \rrbracket=r_{j}, i \leq j \leq n$. Obviously, $\left\langle r_{1}, \ldots, r_{n}\right\rangle \notin$ $I(P, u)$ and $\left\langle r_{1}, \ldots, r_{n}\right\rangle \notin I\left(P, u_{f, k}\right)$, for $k=1,2$, so that $V(A$, $\left.\vec{u}\left[u_{f, k} / i\right], u_{f, k}\right)=0$. Take $u_{f, 1}, u_{f, 1} R v$ iff $v$ is $u_{f, k}$, for $k=1,2$; therefore $V\left(\diamond A, \vec{u}\left[u_{f, 1} / i\right], u_{f, 1}\right)=0$, and, since $u R u_{f, 1}, V(\square \diamond A, \vec{u}, u)=0$.

b. $A$ is $\neg P c_{1}, \ldots, c_{n}, \llbracket c_{j} \rrbracket=r_{j}$. We must distinguish two cases:

i. $P r_{1}, \ldots, r_{n} \in S(u)$. Since $\neg P r_{1}, \ldots, r_{n} \notin S(u),\left\langle r_{1}, \ldots, r_{n}\right\rangle \in$ $I\left(P, u_{f, k}\right), k=1,2$. Thus, $V\left(P c_{1}, \ldots, c_{n}, \vec{u}\left[u_{f, k} / i\right], u_{f, k}\right)=1$, and $V\left(A, \vec{u}\left[u_{f, k} / i\right], u_{f, k}\right)=0$, and, as in $2 \mathrm{a}, V\left(\diamond A, \vec{u}, u_{f, 1}\right)=0$ and $V(\square \diamond A, \vec{u}, u)=0$.

ii. $P r_{1}, \ldots, r_{n} \notin S(u)$. Then, $\left\langle r_{1}, \ldots, r_{n}\right\rangle \in I\left(P, u_{t, k}\right)$ for $k=1,2$, and $V\left(P c_{1}, \ldots, c_{n}, \vec{u}\left[u_{t, k} / i\right], u_{t, k}\right)=1$; therefore $V\left(A, \vec{u}\left[u_{t, k} / i\right]\right.$, $\left.u_{t, k}\right)=0$, and, once more, $V(\square \diamond A, \vec{u}, u)=0$.

Corollary $10 \quad$ Let $N$ be an $\mathbb{L}$ model and let $g(N)$ be as defined above. Then, for any $\vec{u}$ in $W$, any member $v$ of $\vec{u}$ and any wff $A$ of $\mathbb{L}, V(T(A), \vec{u}, v)=1$ iff $\vec{u}, v \vDash A$.

Proof: Follows from Lemmas 7 and 9.

Theorem $11 \quad A$ sentence $A$ is $\mathbb{L}$-valid iff $T(A)$ is $\mathbb{L}(M)$-valid.

Proof: By the definition of $g(N)$, Conditions 1-4 of Lemma 9 are satisfied. By Lemma 9, Condition 5 is satisfied as well. Applying Lemma 9 we obtain the desired result.

Let $N=\langle W, U, D, \mathbb{I} \rrbracket, S\rangle$ be an $\mathbb{L}$-model. We define a binary relation $\subseteq$ in $W$ as follows: for any $u, v \in W, u \subseteq v$ iff $D(u) \subseteq D(v)$ and $S(u) \subseteq S(v)$. We say that a sentence $A$ of $\mathbb{L}$ is positively persistent relative to $i$ iff for any $N, \vec{u}$ in $W$ and $v \in W$ such that $u_{i} \subseteq v$, if $\vec{u} \vDash A$ then $\vec{u}[v / i] \vDash A$. $A$ is negatively persistent relative to $i$ iff for any $N$, $\vec{u}$, and $v \in W$, if $\vec{u} \neq A$ and $u_{i} \subseteq v$ then $\vec{u}$ [ $v / i]=A$. (As in Barba [1], $\vec{u} \vDash A$ abbreviates $\vec{u}, u_{0} \vDash A$, and similarly for =.) $A$ is persistent relative to $i$ iff it is positively and negatively persistent relative to $i$.

Lemma 12 Consider any $\mathbb{L}(M)$-model $M$ and the corresponding $f(M)$. For any $u, v \in \mathbb{W}, u R v$ only if in $f(M), u \subseteq v$.

Proof: We must establish two facts:

1. $D(u) \subseteq D(v)$. This is obvious, since $D(u)=\mathbb{D}(u) \subseteq \mathbb{D}(v)=D(v)$.

2. $S(u) \subseteq S(v) . S(u)=\left\{A: A\right.$ is $(\neg) P r_{1}, \ldots, r_{n}$ and $\left.V(T(A), \vec{u}, u)=1\right\}$. Let $A \in S(u), T(A)$ is $\mathrm{E} ! r_{1} \wedge \ldots \wedge \mathrm{E} ! r_{n} \wedge \square \diamond A$. Since $V(T(A), \vec{u}, u)=1$, $V\left(E ! r_{i}, \vec{u}, u\right)=1,1 \leq i \leq n$ and $V(\square \diamond A, \vec{u}, u)=1$. Take any $v \in \mathbb{W}$, $u R v$. Since domains in $\mathbb{L}(M)$-models are nested, $V\left(E ! r_{i}, \vec{u}[v / j], v\right)=1$ (where $u$ is the $j$ th member of $\vec{u}$ ), and since the propositional basis of our modal system is $S 4, V(\square \square \diamond A, \vec{u}, u)=1$, and so $V(\square \diamond A$, $\vec{u}[v / j], v)=1$. Thus,

$$
V\left(\mathrm{E} ! r_{1} \wedge \ldots \wedge \mathrm{E} ! r_{n} \wedge \square \diamond A, \vec{u}[v / j], v\right)=1,
$$

that is, $V(T(A), \vec{u}[v / j], v)=1$, from which it follows that $A \in S(v)$.

From 1 and 2 conjointly it follows that $u \subseteq v$. 
Theorem 13 A sentence $A$ is positively persistent relative to i iff $T(A) \rightarrow$ $\otimes_{i} \square \otimes_{0} T(A)$ is $\mathbb{L}(M)$-valid, and is negatively persistent relative to $i$ iff $T(\neg A) \rightarrow \otimes_{i} \square \otimes_{0} T(\neg A)$ is $\mathbb{L}(M)$-valid.

Proof:

1. Suppose $A$ is not positively persistent. Then there are $N, \vec{u}, v$ such that $u_{i} \sqsubseteq v$ and $\vec{u}, u_{o} \vDash A$, but $\vec{u}^{\prime}, u_{o}^{\prime} \not A$. Take $g(N)$. Then $V\left(T(A), \vec{u}, u_{0}\right)=1$, $V\left(T(A), \vec{u}^{\prime}, u_{0}^{\prime}\right)=0$, by Corollary 10. This means that $V\left(\otimes_{0} T(A)\right.$, $\left.\vec{u}^{\prime}, v\right)=0$. Since in $g(N), u_{i} R v$ (because $\left.u \sqsubseteq v\right), V\left(\square \otimes_{0} T(A), \vec{u}, u_{i}\right)=0$, so that $V\left(\otimes_{i} \square \otimes_{0} T(A), \vec{u}, u_{0}\right)=0$, and thus $V\left(T(A) \rightarrow \otimes_{i} \square \otimes_{0} T(A)\right.$, $\left.\vec{u}, u_{0}\right)=0$, proving that $T(A) \rightarrow \otimes_{i} \square \otimes_{0} T(A)$ is not valid.

2. Now suppose $T(A) \rightarrow \otimes_{i} \square \otimes_{0} T(A)$ is not valid. Then there are $M, \vec{u}$ in $\mathbb{W}$ and $v \in \mathbb{W}, u_{i} R v$ such that $V\left(T(A), \vec{u}, u_{0}\right)=1$ and $V\left(\otimes_{i} \square \otimes_{0} T(A)\right.$, $\left.\vec{u}, u_{0}\right)=0$, that is, $V\left(\square \otimes_{0} T(A), \vec{u}, u_{i}\right)=0$. Then, there exists $v \in \mathbb{W}$ such that $u_{0} R v$ and $V\left(\otimes_{0} T(A), \vec{u}^{\prime}, v\right)=0$, i.e., $V\left(T(A), \vec{u}^{\prime}, u_{0}^{\prime}\right)=0$. Take $f(M)$. By Corollary $8, \vec{u}, u_{0} \vDash A$ and $\vec{u}^{\prime}, u_{0}^{\prime} \sharp A$. By Lemma 12, $u \sqsubseteq$ $v$, proving that $A$ is not persistent.

For negative persistence, recall that $A$ is positively persistent iff $\neg A$ is negatively persistent, and vice versa.

Acknowledgments - Thanks must be given to the referee for his suggestions and corrections. I am also indebted to Huberto Marraud, without whose advice and encouragement I would never have written this paper (nor any other).

\section{REFERENCES}

[1] Barba, J., "Two formal systems for situation semantics," to appear in Notre Dame Journal of Formal Logic.

[2] Barwise, J. and J. Perry, Situations and Attitudes, The M.I.T. Press, Cambridge, Massachusetts, 1983.

[3] Fitting, M., "Tableau methods of proof for modal logic," Notre Dame Journal of Formal Logic, vol. 13 (1972), pp. 237-247.

[4] Marraud, H., "Tablas analíticas y completud semántica para lógica modal cuantificacional," unpublished.

[5] Marraud, H., “Alternatives to possible worlds semantics," unpublished.

[6] Smullyan, R., First Order Logic, Springer-Verlag, Berlin, Heidelberg, New York, 1968.

[7] Smullyan, R., "A generalization of intuitionistic and modal logics," pp. 274-293 in Truth, Syntax and Modality, edited by H. Leblanc, North-Holland, Amsterdam, 1973.

Departemento de Filosofia

Facultad de Filosofia

Universidad de Valladolid

Plaza de la Universidad

47002-Valladolid, Spain 